\title{
Wir brauchen ein möglichst flexibles Weiterbildungsprogramm
}

\author{
Stefano Bassettia ${ }^{a}$ François Héritier ${ }^{b}$ \\ a Präsident der Weiterbildungskommission der Schweizerischen Gesellschaft für Allgemeine Innere Medizin (SGAIM) \\ ${ }^{b}$ Co-Präsident der Schweizerischen Gesellschaft für Allgemeine Innere Medizin
}

\begin{abstract}
Die Kollegen Dr. Marty [1] und Dr. Sorg [2] werfen in ihren Beiträgen wichtige Fragen auf, welche die SGAIM intensiv beschäftigen. Sie weisen insbesondere auf die Problematik der Nachwuchsförderung und der Attraktivität der hausärztlichen Weiterbildung hin sowie auf mögliche Defizite in der Ausbildung von Hausärzten im Bereich des Bewegungsapparates.
\end{abstract}

Die Problematik ist umfassender und komplexer als in beiden Beiträgen dargestellt: Der internistische Nachwuchs fehlt nicht nur für die Hausarztpraxen, sondern auch für Kaderpositionen in internistischen Kliniken. Und es ist nicht nur die hausärztliche Laufbahn sondern auch die Laufbahn als Allgemeininternist/-in im Spital, welche den jüngeren Kolleginnen und Kollegen offenbar wenig attraktiv erscheint. Es entspricht deshalb nicht der Realität, wenn man behauptet, dass «das neue Weiterbildungsprogramm (WBP) Allgemeine Innere Medizin (AIM) dem ambulanten Sektor Ärzte (entzieht)» [1]. Ebenso realitätsfremd ist es, wenn man behauptet, dass das WBP AIM die Ursache von fehlender interdisziplinärer Kompetenz und Erfahrung in interprofessioneller Zusammenarbeit sei [1], oder wenn man suggeriert, dass das WBP AIM lediglich dazu befähigt, sich mit "exotischen Syndromen» auszukennen, «denen man in einer 30-jährigen Praxiskarriere einmal bis gar nie begegnet». In den letzten 30 Jahren hat sich nicht nur die Tätigkeit in der Praxis, sondern auch die Tätigkeit als Internist im Spital weiterentwickelt, insbesondere auch immer mehr in Richtung einer Team-Arbeit, bei der genau die interdisziplinäre und die interprofessionelle Zusammenarbeit eine ausgesprochen wichtige Rolle haben. Man kann sich auch darüber streiten, welche Krankheitsbilder "exotisch" oder nicht sind - abgesehen davon, dass man diesen naturgemäss nur begegnet, wenn man sie erkennen kann. Man darf sich aber nicht vom Kern des Problems ablenken lassen: Wir sind als Generalisten/-innen in der Praxis und im Spital mit mehreren grossen Herausforderungen konfrontiert. Einerseits scheint heute eine Tätigkeit als Generalist/-in (in der Praxis oder im Spital) weniger attraktiv zu sein, im Vergleich zu einer Tä- tigkeit als Spezialist/-in. Die rasanten Fortschritte der Medizin führen anderseits dazu, dass es heute nicht mehr möglich ist, in allen Gebieten der AIM und der Hausarztmedizin gleich kompetent tätig zu sein. Der «alleswissende» Spitalinternist und die «alleskönnende» Hausärztin, die vielleicht vor 30 Jahren noch existierten, gibt es heute nicht mehr. Ebenso wenig existiert die «durchschnittliche Hausarztpraxis» oder die «durchschnittliche internistische Klinik». Der Hausarzt in einem Tessiner Tal, der Notarzteinsätze bei Ver-

\section{Der internistische Nachwuchs fehlt nicht nur für die Hausarztpraxen.}

kehrsunfällen leistet, wird sich wahrscheinlich besondere zusätzliche Kompetenzen aneignen, die anders sind als die besonderen Kompetenzen einer Hausärztin in Basel, die eine grosse Zahl von HIV-Patienten betreut ... Von allen Seiten werden Defizite in der Weiterbildung moniert, die mittels obligatorischen Kursen (Kommunikation, Ethik, Wirtschaftlichkeit in der Medizin, Palliativmedizin, Umgang mit Antibiotika, Bestimmung der Arbeitsfähigkeit, Versicherungsmedizin, Bestimmung der Fahreignung oder eben die diskutierten Kursen für Traumatologie und Unfallmedizin u.v.a.m.), mittels zusätzlichen obligatorischen Weiterbildungsperioden (x Jahre Chirurgie, Traumatologie, Rheumatologie ...) und mittels obligatorisch zu erfüllenden Zahlen von Untersuchungen oder Eingriffen (x Pleurapunktionen, $x$ Belastungs-EKG usw.) behoben werden sollen. Jede einzelne dieser Forderungen ist isoliert betrachtet wahrscheinlich begründet.

Ein Weiterbildungsprogramm aber, das versuchen sollte, mit vielen Obligatorien und enzyklopädischen 
Listen von geforderten Kenntnissen und Fähigkeiten alle diese Forderungen zu berücksichtigen, wird nicht erfüllbar sein und ein Papiertiger bleiben, der die Qualität der Weiterbildung nicht verbessern wird. Wir brauchen deshalb ein WBP, das möglichst flexibel ist und das erlaubt, dass man neben den Basiskompetenzen modular diejenigen Kompetenzen erwirbt, die

\section{Wir sind als Generalisten in der Praxis und im Spital mit mehreren grossen Herausforde- rungen konfrontiert.}

man für die anvisierte Tätigkeit braucht. Selbstverständlich wird man sich auch nach Erwerb des Facharzttitels kontinuierlich weiterbilden müssen und $\mathrm{Zu}$ satzkompetenzen akquirieren. Es kann aber nicht sein, dass man grundsätzlich zusätzliche obligatorische Zertifikate, Bezeichnungen oder "Labels» erwerben muss, um als Spitalinternistin oder als Hausarzt zu arbeiten. Es muss überlegt werden, ob man das WBP von einem auf Lernzielkatalogen und Obligatorien (Anzahl Untersuchungen, Weiterbildungsperioden) basierendem Programm zumindest teilweise in Richtung Kompetenz-basiertes WBP entwickeln soll, so wie das aktuell auch für das Medizin-Studium in der Schweiz und in anderen Ländern geschieht [3].

Die Weiterbildungskommission der SGAIM plant bereits die Revision der Lernzielkataloge des WBP. Die SGAIM hat zusätzlich eine "Arbeitsgruppe Nachwuchsförderung» eingesetzt, welche im September 2017 fünf Empfehlungen abgegeben hat, die von verschiedenen Gremien der SGAIM weiter verfolgt werden:

1. Revision der Lernzielkataloge des WBP,

2. Aufbau von systematischen, koordinierten Weiterbildungs-Curricula (u.a. mit systematischem Mentoring und Karriereberatung und, zumindest in grösseren Spitälern, spezifischen Karrieretracks Hausarzt und Spitalinternist),

3. "More action, less administration!»: vermehrter Akzent der Weiterbildung auf Akutmedizin (Notfall), systematische Vermittlung von sinnvollen technischen Fertigkeiten (z.B. Ultraschall, Punktionen), Reduktion von administrativen Tätigkeiten durch Einsatz von Supportfunktionen («Care Koordinatorinnen» u.s.w.),

4. Gezielte Förderung der internistischen Forschung und Entwicklung sowie der Lehre,

5. Gezielte Schaffung eines positiven Rollenbilds des Allgemeininternisten.

Die entscheidende Rolle der Allgemeininternisten im Spital und in der Praxis, die in der Lage sind, ihre $\mathrm{Pa}$ tienten holistisch $\mathrm{zu}$ betreuen und somit wirklich "personalisierte Medizin" zu betreiben - im Gegensatz

Die Weiterbildungskommission der SGAIM plant bereits die Revision der Lernzielkataloge des WBP.

zu einer Organ-zentrierten, fragmentierten Medizin [4] - wird auch international zunehmend wiederentdeckt. Unser WBP AIM muss dieser Rolle gerecht werden und die Basis sein für eine erfolgreiche Tätigkeit als Generalist/-in in der Praxis oder im Spital.

Literatur

1 Marty F. Sechs Jahre Weiterbildungsprogramm «Allgemeine Innere Medizin». Ein Feedback aus der Praxis. Schweiz Ärztezeitung. 2018;99(11):353-5.

2 Sorg B. Defizite in der Ausbildung von Hausärztinnen und Hausärzten. Fehlende Kompetenz am Bewegungsapparat. Schweiz Ärztezeitung. 2018;99(11):356

3 Powell DE, Carraccio C. Toward competency-based medical education. N Engl J Med. 2018;378:3-5.

4 Greene JA, Loscalzo J. Putting the patient together-social medicine, network medicine, and the limits of reductionism. N Engl J Med. 2017;2493-9. 